\title{
Grooved pegboard test performance before and after cerebrospinal fluid tap test in patients with idiopathic normal pressure hydrocephalus
}

\section{Caiyan Liu}

Peking Union Medical College Hospital https://orcid.org/0000-0001-6035-7456

\section{Liling Dong}

Peking Union Medical College Hospital

\section{Chenhui Mao}

Peking Union Medical College Hospital

Jie Li

Peking Union Medical College Hospital

\section{Xinying Huang}

Peking Union Medical College Hospital

Junji Wei

Peking Union Medical College Hospital

\section{Bo Hou}

Peking Union Medical College Hospital

\section{Feng Feng}

Peking Union Medical College Hospital

\section{Liying Cui}

Peking Union Medical College Hospital

\section{Jing Gao ( $\sim$ gj107@163.com )}

Peking Union Medical College Hospital, Chinese Academy of Medical Sciences

\section{Research article}

Keywords: Idiopathic normal pressure hydrocephalus, cerebrospinal fluid tap test, grooved pegboard test

Posted Date: January 15th, 2021

DOl: https://doi.org/10.21203/rs.3.rs-145937/v1

License: (c) (1) This work is licensed under a Creative Commons Attribution 4.0 International License. Read Full License 


\section{Abstract}

\section{Introduction}

Motor impairment in patients with idiopathic normal pressure hydrocephalus (iNPH) can extend beyond gait and include deficits in upper extremity functions and psychomotor speed. Evaluation of upper extremity function will be helpful for iNPH patients who are unable to ambulate (e.g., wheelchair-bound patients) and may not be able to comply with the gait evaluation. Our study aimed to explore the use of the grooved pegboard test (GPT) to assess responsiveness to the cerebrospinal fluid (CSF) tap test (TT) in iNPH patients.

\section{Methods}

Sixty-five possible NPH patients were enrolled retrospectively and all underwent detailed neuropsychological and walking assessments, CSF TTs, and brain magnetic resonance imaging. The GPT results before and after the CSF TT were compared and correlated with the other clinical assessments. In diffusion tensor imaging analysis, the fractional anisotropy (FA) and mean diffusivity (MD) values of periventricular white matter were measured by the region of interest method and were correlated with pegboard test performance.

\section{Results}

Compared with 0-hour GPT-dominant and nondominant hand performance, GPT-dominant hand and nondominant hand performance on the 24-hour and 72-hour CSF TT were significantly improved (all $\mathrm{P}<$ 0.01). There were no statistically significant differences between 24-hour and 72-hour dominant hand GPT performance. The improvement ratios in the complex visual motor speed index (i.e., the GPT performance combined with the symbol-digit modalities test score) were significantly different between the CSF TT responder and nonresponder groups. The baseline GPT performance correlated with walking test, cognitive function, and functional scores. The diffusion tensor imaging analysis of eighteen patients showed that bilateral GPT performance was significantly correlated with FA values in right periventricular lesions (both $\mathrm{P}<$ 0.05).

\section{Conclusion}

GPT could measure the change of upper extremity motor function after TT and correlated the lower extremity motor function. It was worth further investigation to its application for iNPH patients unable to comply with the gait evaluation.

\section{Background}

Idiopathic normal pressure hydrocephalus (iNPH) patients present disturbances in gait and balance, cognition and/or control of urination with neuroimaging characterized by enlargement of the cerebral ventricles [1-3]. Typically, gait disturbance in iNPH patients is the initial and most prominent symptom [4, 5], but motor impairments can also extend to include upper extremity dysfunction [6-9]. Cognitive impairment in iNPH patients is generally characterized by frontal system deficits that may include slowing of information 
processing, task shifting, reduced learning and executive abilities [10-12]. Bladder control dysfunction can range from increased urinary urgency and frequency to frank incontinence [13].

A commonly used auxiliary test for predicting shunt responsiveness is the cerebrospinal fluid (CSF) tap test (TT), a procedure in which iNPH symptoms are assessed before and after drainage of $30-50 \mathrm{~cm} 3$ of CSF by lumbar puncture. Clinical improvement following lumbar puncture indicates an increased likelihood of improvement after shunt placement [14-16]; however, a negative CSF TT does not rule out shunt responsiveness [17]. Standard methods for determining the CSF TT response are based on the clinical impression of changes in gait and cognition after lumber puncture. Instruments to evaluate gait $[18,19]$ can be useful in this assessment but are not always sensitive to subtle changes. iNPH patients who are unable to ambulate (e.g., the patient is wheelchair bound) may not be able to comply with the gait evaluation but still benefit from shunt placement. In mildly affected patients, testing may not reveal sufficiently profound impairments to permit assessment of change after a lumber puncture. Therefore, we sought to identify additional measures that can be used to objectively assess responsiveness to the CSF TT.

The grooved pegboard test (GPT) assesses eye-hand coordination and motor speed and thus requires sensory-motor integration and a high level of motor processing [20]. It is considered a more complex motor task than others, such as grip strength or finger tapping. As such, it requires more effort and is more sensitive to psychomotor speed [21]. In iNPH patients, some researchers have used GPT performance as a psychometric measure for upper extremity motor and psychomotor speed [22]. Upper extremity motor tests may be particularly advantageous in situations where the severity of impairment precludes accurate and reliable assessments of changes in gait. According to the literature[22] and our clinical experience, the complex visual motor speed index (a combined measure of GPT and symbol-digit modalities test performance) may be as useful as GPT for the assessment of iNPH patients.

Herein, we performed a pilot study to investigate changes in GPT performance before and after CSF TT. The improvement ratio of the GPT performance and that of the complex visual motor speed index were compared between the CSF TT responder and nonresponder groups. We explored whether GPT performance correlated with clinical parameters and white matter lesions.

\section{Methods}

\section{Patients, history taking and clinical assessment}

We retrospectively reviewed patients with possible iNPH who were admitted to the neurological or neurosurgery department ward of Peking Union Medical College Hospital for CSF TT. All patients were diagnosed according to the guidelines for the clinical diagnosis of idiopathic NPH (iNPH) published in 2005 [23] and the Chinese consensus for iNPH [24].

In the CSF TT and iNPH registry protocol, age, sex, past histories, personal histories, and initial and full-blown symptoms for all patients were recorded. Patients also completed the Mini-Mental State Examination (MMSE) [25], Montreal Cognitive Assessment (MOCA) [26], and activities of daily living (ADL) questionnaire [27]. They also underwent a brief neuropsychological battery assessment including the Symbol-Digit 
Modalities Test [28], Trail Making Test A [29] and the Stroop Color Word Task-C [30]. The iNPH Grading Scale (iNPHGS) [31] was used to rate the severity of each fundamental symptom of iNPH (gait disturbance, cognitive impairment, and urinary incontinence) on a four-point scale after a detailed interview with the patients and caregivers. All subjects underwent a CSF TT and brain MRI.

The CSF TT was performed by lumbar puncture followed by the measurement of CSF opening pressure and drainage of $30 \mathrm{ml}$ of CSF. Before and after the procedure, cognitive function and walking ability were evaluated by the means of the 5-meter up and go test (TUG), the 10-meter walking test, the GPT(Model 32025, Lafayette instrument, USA)and a brief executive function battery, which was previously described [32]. The time of TUG, the time and steps that the patient took to complete the 10-meter walking test and a video of the walking tests were recorded. Additional evaluations were conducted at 8 hours, 24 hours and 72 hours after CSF TT. The following criteria were used to identify responders: 1. CSF TT responders were defined as patients with reductions in their time and number of steps in the walking test at least once after the CSF TT, with decreases of $10 \%$ for both parameters or a decrease of $20 \%$ on at least one of these parameters [33]. 2 . Gait was improved based on videos that were evaluated by two neurologists who were blinded to the patient's clinical and neuroimaging characteristics. The patients who met 1 of 2 criteria were identified as CSF TT responders.

After the detailed clinical assessment, the patients with neuroimaging support or CSF TT response were suggested to have shunting.

\section{Inclusion and exclusion criteria}

The patients were recruited in the study according to the following criteria: 1 . the patients completed the multi-time point assessment of walking and neuropsychological tests; 2 . the patients had undergone at least 3 GPT sessions, including a baseline session. The exclusion criteria were as follows: 1. patients who were unable to finish GPT for any reason; 2. patients who could not tolerate $30 \mathrm{ml}$ of CSF drainage during the CSF TT.

\section{Brain MRI}

Axial and sagittal spin-echo T1-weighted images, axial fast spin-echo T2-weighted, fluid-attenuated inversion recovery images were obtained by using a 1.5-T MRI unit (Signa Excite, General Electric, Milwaukee, WI, USA). Diffusion tensor imaging (DTI) was one selected test for patients. We obtained and analyzed 18 patients' DTI data. The regions of interest (ROIs) were the bilateral anterior and posterior periventricular white matter that were set as circular areas with a diameter of $2 \mathrm{~mm}$ perpendicular to the longitudinal axis of the ipsilateral ventricle on the brain imaging slice with the lateral ventricle (detailed in Fig. 1). The fractional anisotropy (FA) and mean diffusivity (MD) values were measured across ROIs on DTI images. The ROIs were chosen according to our pilot study data [34], which indicated that the FA and MD values in the bilateral anterior and posterior periventricular white matter were correlated with walking ability and cognition in possible iNPH patients. A consistency test was performed (Cronbach's a coefficient $>0.6$ ).

\section{Statistical methods}


The continuous variables were described as mean \pm standard deviation or median and interquartile range, as appropriate. The scores for various evaluation indexes before and 8 hours, 24 hours and 72 hours after CSF TT conformed to skewed distributions. The differences in the GPT performance among the different time points before and after the CSF TT (i.e., baseline, 8 hours, 24 hours and 72 hours) were analyzed using a nonparametric paired sign rank-sum test. The Bonferroni correction was applied for the repeated measurements, and the statistical significance level was set at $p<0.05 / 4=0.01$.

We compared the maximum improvement ratios for the GPT performance between the CSF TT responder and nonresponder groups by means of the Mann-Whitney $U$ test. The improvement ratio was calculated as follows: (baseline GPT performance - GPT performance after CSF TT)/baseline GPT performance. The maximum improvement ratio for GPT was extracted for the different times (i.e., 8 hours, 24 hours, and 72 hours) after the CSF TT.

The GPT and symbol-digit modalities test score were combined into the complex visual motor speed index. The maximum improvement ratios for the complex visual motor speed index after CSF TT were also compared between the responder and nonresponder groups using the Mann-Whitney $U$ test. For 8 hours, 24 hours, and 72 hours after CSF TT, we also correlated the improvement ratio of the GPT performance on each time point with that of the corresponding walking test and the improvement ratio of the complex visual motor speed index on each time point with that of the walking test by means of Spearman's correlation. According to the literature reported[22], The formula was the improvement ratio of the complex visual motor speed index $=$ (the improvement ratio of symbol-digit modalities test raw score + the improvement ratio of mean bilateral GPT performance)/2. The statistical significance level was set at $p<0.05$.

The correlations between the baseline GPT results and the baseline clinical assessments, including iNPHGS scores, walking test results, and neuropsychological performance, were tested by means of Spearman's correlations. The correlations between the baseline GPT results and the DTI parameters from the periventricular white matter lesions were also analyzed using Spearman's correlation method. The statistical significance level was set at $p<0.05$.

All statistical analyses were performed with the statistical software package SPSS for Windows (version 13.0.; SPSS Inc., Chicago, IL, USA).

\section{Results}

\section{Demographic characteristics of CSF TT responders and nonresponders}

Sixty-five iNPH patients were recruited for this study. According to our criteria, there were 29 CSF TT responders and 36 nonresponders. There were no significant differences in age, sex, disease duration, iNPHGS, MMSE, MOCA scores and 10-meter walking time, but there was a difference in the ADL scores (Table 1) between the CSF TT responder and nonresponder groups. 
Table 1

Demographic characteristics of the CSF TT responder and nonresponder groups

\begin{tabular}{|llll|}
\hline & Responders $(\mathbf{n}=\mathbf{3 6})$ & Nonresponders $(\mathbf{n = 2 9 )}$ & P value \\
\hline Age (years old) & $69 \pm 11$ & $68 \pm 9$ & 0.74 \\
\hline Gender (male:female) & $30: 6$ & $22: 7$ & 0.54 \\
\hline Duration (years) & $4.2 \pm 3.4$ & $3.9 \pm 2.8$ & 0.74 \\
\hline iNPHGS total & $5.7 \pm 1.7$ & $5.2 \pm 2.1$ & 0.26 \\
\hline MMSE & $20.8 \pm 7.0$ & $23.9 \pm 5.6$ & 0.14 \\
\hline MOCA & $17.6 \pm 7.3$ & $19.1 \pm 6.2$ & 0.50 \\
\hline ADL & $40.7 \pm 14.1$ & $30.0 \pm 9.5$ & $0.02 *$ \\
\hline 10-meter walking time (seconds) & $23.6 \pm 30.6$ & $41.8 \pm 136.5$ & 0.44 \\
\hline *P<0.05 & & & \\
\hline
\end{tabular}

\section{Grooved pegboard test performance changes from before to after the CSF TT}

The bilateral GPT results at 24 hours and 72 hours after the CSF TT were significantly improved compared with those before the CSF TT $(P<0.01)$ (Table 2). We also tested the GPT result differences at 8 hours, 24 hours and 72 hours after the CSF TT. There were significant differences among the three time points with the nondominant hand. With the dominant hand, there were significant differences at 8 hours vs. 72 hours and at 24 hours vs. 72 hours after CSF TT. (Table 2) 
Table 2

Comparison of the grooved pegboard test (GPT) performance[median(inter quartile range)] before and after the CSF TT in sixty-five patients with iNPH

\begin{tabular}{|c|c|c|c|c|c|c|}
\hline & $\begin{array}{l}\text { GPT-nondominant } \\
\text { hand[median(interquartile } \\
\text { range)] }\end{array}$ & $\begin{array}{l}\text { Wil- } \\
\text { coxon } \\
\text { Z }\end{array}$ & $\mathbf{P}$ & $\begin{array}{l}\text { GPT-dominant } \\
\text { hand[median(interquartile } \\
\text { range)] }\end{array}$ & $\begin{array}{l}\text { Wil- } \\
\text { coxon } \\
\mathrm{Z}\end{array}$ & $\mathbf{P}$ \\
\hline Baseline & $137.0(103.8,226.0)$ & & & $121.5(97.3,202.3)$ & & \\
\hline $\begin{array}{l}8 \mathrm{hr} \\
\text { after } \\
\text { CSF TT }\end{array}$ & $134.5(96.5,231.5)$ & $-1.11^{1}$ & $0.27^{1}$ & $122.0(93.6,177.3)$ & $-2.24^{4}$ & $0.03^{4}$ \\
\hline \multirow[t]{2}{*}{$\begin{array}{l}24 \mathrm{hr} \\
\text { after } \\
\text { CSF TT }\end{array}$} & $127.0(94.3,207.6)$ & $-4.47^{1}$ & $\begin{array}{l}< \\
0.01^{1 *}\end{array}$ & $119.0(86.8,181.5)$ & $-3.48^{4}$ & $<.01^{4 *}$ \\
\hline & & $-2.91^{2}$ & $\begin{array}{l}< \\
0.01^{2 *}\end{array}$ & & $-2.61^{5}$ & $\begin{array}{l}< \\
0.01 * 5\end{array}$ \\
\hline \multirow[t]{3}{*}{$\begin{array}{l}72 \mathrm{hr} \\
\text { after } \\
\text { CSF TT }\end{array}$} & $119.0(93,181.6)$ & $-5.22^{1}$ & $\begin{array}{l}< \\
0.01^{1 *}\end{array}$ & $112.6(89.0,174.0)$ & $-3.87^{4}$ & $\begin{array}{l}<.01^{4 *} \\
\text { t }\end{array}$ \\
\hline & & $-4.96^{2}$ & $\begin{array}{l}<.01^{2 *} \\
\text { t }\end{array}$ & & $-3.42^{5}$ & $\begin{array}{l}<.01^{5 *} \\
\text { t }\end{array}$ \\
\hline & & $-4.10^{3}$ & $\begin{array}{l}<.01^{3 *} \\
\end{array}$ & & $-2.05^{6}$ & $0.04^{6}$ \\
\hline \multicolumn{7}{|c|}{ Note: ${ }^{*} \mathrm{P}<0.01$} \\
\hline \multicolumn{7}{|c|}{${ }^{1}$ compared to the baseline GPT result with the nondominant hand } \\
\hline \multicolumn{7}{|c|}{${ }^{2}$ compared to the GPT result with the nondominant hand at $8 \mathrm{hr}$ after the CSF TT } \\
\hline \multicolumn{7}{|c|}{${ }^{3}$ compared to the GPT result $24 \mathrm{hr}$ after the CSF TT } \\
\hline \multicolumn{7}{|c|}{${ }^{4}$ compared to the baseline GPT result with the dominant hand } \\
\hline \multicolumn{7}{|c|}{${ }^{5}$ compared to the GPT result with the dominant hand $8 \mathrm{hr}$ after the CSF TT } \\
\hline${ }^{6}$ compar & $d$ to the GPT result with the $c$ & minant & and $24 \mathrm{r}$ & after the CSF TT & & \\
\hline
\end{tabular}

Comparison of the maximum improvement ratios for the GPT, symbol-digit modalities test, and complex visual motor speed index between the CSF TT responder and nonresponder groups

There was a significant difference in the maximum improvement ratio of the complex visual motor speed index (combined GPT with symbol-digit modalities) between the CSF TT responder and nonresponder groups [median (interquartile range) was $0.20(0.15,0.43)$ vs. $0.12(0.02,0.31), \mathrm{U}=256.5, \mathrm{P}=0.04$ ]. The maximum improvement ratios for the GPT were not significantly different between the two groups [median (interquartile range) was $0.24(0.14,0.32)$ vs. $0.19(0.11,0.36), \mathrm{U}=432, \mathrm{P}=0.24]$. The maximum improvement ratios for the 
symbol-digit modalities test were also not different [median (interquartile range) were $0.31(0.16,0.41)$ vs. $0.14(0,0.46), U=292.5, P=0.10]$. (Fig. 2)

Correlations of the improvement ratio of the grooved pegboard test and the complex visual motor speed index after the CSF TT with the improvement ratio of the walking test

We also correlated the improvement ratio of the GPT performance and the complex visual motor speed index post CSF TT with the improvement ratio of the TUG, 10-meter walking time and steps on the same evaluation time. The results showed that the improvement ratio of the GPT performance 24 hours after the CSF TT was significantly correlated with the 10 meter walking step improvement ratio at the corresponding time point. $(r=$ $0.27, p=0.03$ ). The improvement ratio of the GPT performance 72 hours after the CSF TT was borderline correlated with the TUG time improvement ratio $(r=0.25, p=0.05)$. The improvement ratio of the complex visual motor speed index 72 hours after the CSF TT was significantly correlated with the TUG time improvement ratio $(r=0.32, p=0.02)$ and borderline correlated with the 10 meter walking step improvement ratio $(r=0.27, p=0.05)$.

\section{Correlations between baseline grooved pegboard test performance and clinical parameters}

The baseline mean bilateral GPT results were significantly correlated with the baseline INPHGS total, INPHGS walking, MMSE, MOCA and ADL scores and 10-meter walking test times $(r=0.36,0.46,-0.50,-0.49,0.66,0.32$, respectively, all $P<0.01)$ and were not correlated with INPHGS cognition $(r=0.25, P=0.05)$ or urinary score $(r$ $=0.16, P=0.21)$.

Correlations between baseline grooved pegboard test performance and periventricular white matter lesion DTI parameters in possible iNPH patients

Eighteen patients underwent DTI evaluation. The bilateral GPT performance correlated with the right periventricular anterior horn FA values (right: $r=-0.48, P=0.04$, left: $r=-0.55, P=0.02$ ). The right GPT results correlated with the left periventricular anterior horn MD values $(r=0.49, P=0.04)$. There were no significant correlations between the GPT results and periventricular posterior horn FA and MD values.(Table 3) 
Table 3

Correlation between grooved pegboard test (GPT) performance of possible iNPH patients and the parameters of periventricular white matter lesions by means of diffusion tensor imaging

\begin{tabular}{|c|c|c|c|c|}
\hline & $\begin{array}{l}\text { Right GPT } \\
\text { performance } \\
(n=18)\end{array}$ & & $\begin{array}{l}\text { Left GPT } \\
\text { performance } \\
(n=18)\end{array}$ & \\
\hline & Rho & $P$ & Rho & $P$ \\
\hline $\begin{array}{l}\text { Right periventricular -anterior horn FA } \\
\text { value }\end{array}$ & -0.48 & $0.04^{\star}$ & -0.55 & $0.02^{*}$ \\
\hline $\begin{array}{l}\text { Right periventricular -anterior horn MD } \\
\text { value }\end{array}$ & 0.27 & 0.28 & 0.18 & 0.47 \\
\hline $\begin{array}{l}\text { Left periventricular-anterior horn FA } \\
\text { value }\end{array}$ & -0.40 & 0.09 & -0.42 & 0.08 \\
\hline $\begin{array}{l}\text { Left periventricular -anterior horn MD } \\
\text { value }\end{array}$ & 0.49 & $0.04^{\star}$ & 0.44 & 0.07 \\
\hline $\begin{array}{l}\text { Right periventricular -posterior horn FA } \\
\text { value }\end{array}$ & -0.21 & 0.40 & -0.20 & 0.43 \\
\hline $\begin{array}{l}\text { Right periventricular -posterior horn MD } \\
\text { value }\end{array}$ & 0.08 & 0.75 & 0.05 & 0.85 \\
\hline $\begin{array}{l}\text { Left periventricular -posterior horn FA } \\
\text { value }\end{array}$ & -0.37 & 0.14 & -0.39 & 0.12 \\
\hline $\begin{array}{l}\text { Left periventricular -posterior horn MD } \\
\text { value }\end{array}$ & 0.16 & 0.54 & 0.12 & 0.65 \\
\hline \multicolumn{5}{|c|}{ FA fractional anisotropy, MD mean diffusivity } \\
\hline$\star P<0.05$ & & & & \\
\hline
\end{tabular}

\section{Discussion}

The present findings demonstrate that upper extremity motor functions can improve following TT, providing an additional measure of a clinical response. The GPT results were correlated with cognition and lower extremity motor function. The improvement ratios for the complex visual motor speed index (the GPT performance combined with the symbol-digit modalities test performance) were significantly different between the CSF TT responder and nonresponder groups. Additionally, the bilateral GPT results were correlated with periventricular anterior horn white matter lesion FA values.

Upper extremity function is impacted in iNPH patients, although lower extremity motor function is the primary concern in iNPH patients. There have been several studies on upper extremity motor function after CSF drainage tests and shunting operations in iNPH patients. Tsakanikas $D$ et al reported that these upper extremity motor tests may be useful as sensitive markers of change after shunt placement in iNPH patients (e.g., line tracing tasks) [22]. Kang et al reported that upper limb motor function was improved after CSF TT and shunting and was correlated with gait parameters [7]. Liouta E et al also found that finger tapping 
improvements after CSF TT were useful for the differential diagnosis and prediction of shunt treatment outcomes [35]. The strength of our study is the multiple time point assessments of upper extremity motor function. We also found that the complex visual motor speed index (a combined measure of GPT and symbol-digit modalities test performance) was more useful in demonstrating significant differences between the CSF TT responder and nonresponder groups. The complex visual motor speed index might be a promising candidate measure to recognize CSF TT responders. Tsakanikas D mentioned that there was no significant decrease in the complex visual motor speed index after CSF TT [22]. However, they performed the evaluation just once, at 2-4 hours after the CSF TT, which might have missed the time point showing the best improvement.

Our study also provided evidence of the correlations among GPT performance and cognition and walking ability in patients with iNPH. The correlation of walking and executive neurocognitive deficits has been reported in iNPH patients and supports the notion that gait deficits are due to executive motor planning dysfunction [36]. Upper extremity motor function seems to be consistent with changes in walking ability and cognition, which indicates common mechanisms of motor disturbance and cognitive impairment in iNPH patients. This relationship also supported the notion that the GPT can be a reliable and useful assessment tool for iNPH patients.

The correlation of bilateral GPT results with the FA value of the anterior periventricular horn white matter lesions provided mechanistic evidence of upper limb motor dysfunction in iNPH patients. White matter lesions are very common in iNPH patients. The reduction in the irregular type of periventricular hyperintensity located around the frontal horns after surgery has been reported to be associated with clinical improvements in patients with iNPH [37]. Our study results showed that in the DTI analysis, the GPT results for both hands were correlated with FA values in white matter lesions in the anterior periventricular horn. We speculated that the upper extremity motor improvement in patients with iNPH after CSF TT drainage was related to the periventricular hyperintensity located around the frontal horns and might be reversible through shunting.

Our study has several limitations. First, the sample size of patients undergoing surgery was small, and the predictive effect of the GPT performance on the shunting outcome was not investigated. Second, only eighteen patients underwent DTI analysis. Third, this is a pilot and retrospective study, and GPT performance was analyzed among patients with iNPH who were able to walk and complete multiple time point evaluations after CSF TT. The application of the GPT in patients who are unable to ambulate needs to be explored in the future.

\section{Conclusions}

Our study reported that performance on the GPT was related to lower extremity motor ability. The improvement ratios for the complex visual motor speed index (the GPT performance combined with the symbol-digit modalities test performance) were significantly different between the CSF TT responder and nonresponder groups. The upper extremity motor function tests should be investigated further and might be an alternative evaluation tool for patients who are unable to ambulate and may not be able to comply with the gait evaluation. 


\section{Abbreviations}

iNPH: Idiopathic normal pressure hydrocephalus; CSF: Cerebrospinal fluid; TT: Tap test; GPT: Grooved pegboard test; MMSE: Mini-Mental State Examination; MOCA: Montreal Cognitive Assessment; ADL: Activities of daily living; iNPHGS: iNPH Grading Scale; TUG: 5-meter up and go test; DTI: Diffusion tensor imaging; ROIs: The regions of interest; FA: Fractional anisotropy; MD: Mean diffusivity.

\section{Declarations}

\section{Ethics approval and consent to participate}

Permission for the research was received from the Research Ethics Board of the Peking Union Medical College Hospital (PUMCH). Written informed consent was obtained from all patients participating in the study.

\section{Consent for publication}

Each of the authors has read and concurs with the content in the final manuscript.

\section{Availability of data and materials}

The data sets in this study are available from the corresponding author on reasonable request.

\section{Competing interests}

The authors declare that they have no conflicts of interest/competing interests.

\section{Funding}

This study was supported by a grant from CAMS 2016-12M-1-004, National NSFC81550021.

\section{Authors' contributions}

Caiyan Liu and Jing Gao drafted and revised the manuscript, contributed to the data analysis and interpreted the results. The study was designed by Caiyan Liu and Jing Gao. Study supervision was performed and study funding was obtained by Jing Gao and Liying Cui. Caiyan Liu, Liling Dong, Chenhui Mao, Jie Li, and Xinying Huang contributed to the clinical assessment and data acquisition. Bo Hou and Feng were responsible for the neuroimaging data collection and analysis. Junji Wei contributed to the neurosurgery treatment for patients. Caiyan Liu performed the statistical analysis. All authors read and approved the final manuscript.

\section{Acknowledgements}

We would like to thank all the patients and caregivers who participant in the study.

\section{References}


1. Adams RD, Fisher CM, Hakim S, et al. Symptomatic occult hydrocephalus with "normal" cerebrospinalfluid pressure a treatable syndrome. N Engl J Med.1965;273:117-26.

2. Graff-Radford NR, Jones DT. Normal Pressure Hydrocephalus. Continuum (Minneap Minn). 2019;25(1):165-186. doi: 10.1212/CON.0000000000000689.

3. Isaacs AM, Williams MA, Hamilton MG. Current Update on Treatment Strategies for Idiopathic Normal Pressure Hydrocephalus. Curr Treat Options Neurol. 2019;3;21(12):65. doi: 10.1007/s11940-019-0604-z.

4. Meier U, Zeilinger FS, Kintzel D. Signs, symptoms and course of normal pressure hydrocephalus in comparison with cerebral atrophy. Acta Neurochir (Wien). 1999;141(10):1039-48.doi: $10.1007 /$ s007010050480

5. Palm WM, Saczynski JS, van der Grond J, et al. Ventricular dilation: association with gait and cognition.Ann Neurol. 2009;66(4):485-93. doi: 10.1002/ana.21739.

6. Blomsterwall E, Bilting M, Stephensen $\mathrm{H}$, et al. Gait abnormality is not the only motor disturbance in normal pressure hydrocephalus. Scand J Rehabil Med. 1995;27:205-9.

7. Kang K, Jeon JS, Kim T, et al. Asymmetric and Upper Body Parkinsonism in Patients with Idiopathic Normal-Pressure Hydrocephalus.J Clin Neurol. 2016;12(4):452-459. doi: 10.3988/jen.2016.12.4.452.

8. Molde K, Söderström L, Laurell K. Parkinsonian symptoms in normal pressure hydrocephalus: a population-based study.J Neurol. 2017;264(10):2141-2148. doi: 10.1007/s00415-017-8598-5.

9. Mandir AS, Hilfiker J, Thomas G, et al.Extrapyramidal signs in normal pressure hydrocephalus: an objective assessment. Cerebrospinal Fluid Res. 2007;13:4-7. doi: 10.1186/1743-8454-4-7

10. Iddon JL, Pickard JD, Cross JJ, et al. Specific patterns of cognitive impairment in patients with idiopathic normal pressure hydrocephalus and Alzheimer's disease: a pilot study. J Neurol Neurosurg Psychiatry. 1999;67(6):723-32. doi:10.1136/jnnp.67.6.723

11. Klinge P, Ruckert N, Schuhmann M, et al. Neuropsychological testing to improve surgical management of patients with chronic hydrocephalus after shunt treatment. Acta Neurochir. 2002;Suppl 81:51-3. doi:10.1007/978-3-7091-6738-0_13

12. Kanno S1, Saito M, Hayashi A, et al. Counting-backward test for executive function in idiopathic normal pressure hydrocephalus. Acta Neurol Scand. 2012;126(4):279-86. doi: 10.1111/j.16000404.2012.01644.x.

13. Sakakibara R, Kanda T, Sekido T, et al. Mechanism of bladder dysfunction in idiopathic normal pressure hydrocephalus. Neurourol Urodyn. 2008;27(6):507-510. doi: 10.1002/nau.20547.

14. Damasceno BP, Carelli EF, Honorato DC, et al. The predictive value of cerebrospinal fluid tap-test in normal pressure hydrocephalus. Arq Neuropsiquiatr. 1997;55(2):179-85. doi:10.1590/s0004$282 \times 1997000200003$

15. Wikkelso $\mathrm{C}$, Andersson $\mathrm{H}$, Blomstrand $\mathrm{C}$, et al. The clinical effect of lumbar puncture in normal pressure hydrocephalus. J Neurol Neurosurg Psychiatry. 1982;45(1):64-9. doi:10.1136/jnnp.45.1.64

16. Mihalj M, Dolić K, Kolić K, et al. CSF tap test - Obsolete or appropriate test for predicting shunt responsiveness? A systemic review. J Neurol Sci. 2016;15(362):78-84. doi: 10.1016/j.jns.2016.01.028.

17. Boon AJ, Tans JT, Delwel EJ, et al. Dutch normal-pressure hydrocephalus study: prediction of outcome after shunting by resistance to outflow of cerebrospinal fluid. J Neurosurg.1997;87(5):687-93. 
doi:10.3171/jns.1997.87.5.0687

18. Malm J, Kristensen B, Karlsson T, et al. The predictive value of cerebrospinal fluid dynamic tests in patients with idiopathic adult hydrocephalus syndrome. Arch Neurol. 1995;52(8):783-9. doi:10.1001/archneur.1995.00540320059013

19. Ravdin LD, Katzen HL, Jackson AE, et al. Features of gait most responsive to tap test in normal pressure hydrocephalus. Clin Neurol Neurosurg. 2008;110(5):455-61. doi:10.1016/j.clineuro.2008.02.003

20. Sage MD, Bryden PJ, Roy EA, et al. The relationship between the grooved pegboard test and clinical motor symptom evaluation across the spectrum of Parkinson's disease severity.J Parkinsons Dis. 2012;2(3):207-13. doi: 10.3233/JPD-2012-012093.

21. Mitrushina M1, Fogel T, D'Elia L, et al. Performance on motor tasks as an indication of increased behavioral asymmetry with advancing age. Neuropsychologia. 1995;33(3):359-64. doi:10.1016/00283932(94)00113-4.

22. Tsakanikas D, Katzen H, Ravdin LD, et al. Upper extremity motor measures of Tap Test response in Normal Pressure Hydrocephalus.Clin Neurol Neurosurg. 2009;111(9):752-7. doi: 10.1016/j.clineuro.2009.07.017.

23. Marmarou A, Bergsneider M, Relkin N, et al. Development of guidelines for idiopathic normal-pressure hydrocephalus: introduction.Neurosurgery. 2005;57(3 Suppl):S1-3; discussion ii-v. doi:10.1227/01.neu.0000168188.25559.0e

24. Wei JJ, Hu J, Gao J, et al. [Expert consensus on diagnosis and treatment of idiopathic normal pressure hydrocephalus in China]. Chinese Medical Journal. 2016;96(21):1635-1638.(in Chinese)

25. Folstein MF, Folstein SE, McHugh PR. "Mini-Mental State” a practical method for grading the cognitive state of patients for the clinician. J Psychiatr Res. 1975;12(3):189-98.

26. Tan JP, Li N, Gao J, et al. Optimal cutoff scores for dementia and mild cognitive impairment of the Montreal Cognitive Assessment among elderly and oldest-old Chinese population. Journal of Alzheimer's disease. 2015;43(4):1403-12. doi:10.3233/jad-141278.

27. Zhang Ming-yuan, Elena Yu, He Yan-ling. [Questionnaire and application of daily living ability scale]. Shanghai psychiatry. 1995; (supplementary): 5-6.(in Chinese区

28. Sheridan LK, Fitzgerald HE, Adams KM, et al.Normative Symbol Digit Modalities Test performance in acommunity-based sample. Archives of Clinical Neuropsychology. 2006;21:23-8.

29. Lu JC, Guo QH, Hong Z, et al.[Trail Making Test Used by Chinese Elder ly Patients with Mild Cognitive Impairment and Mild Alzheimer Dementia].Chinese Journal of Clinical Psychology. 2006;14(2):118-120. (in Chinese)

30. Guo QH, Hong Z, Lv CZ, et al.[Application of Stroop color-word test on Chinese elderly patients with mild cognitive impairment and mild Alzheimer's dementia]. Chin J Neuromed, 2005;4:701-4. (in Chinese)

31. Kubo Y1, Kazui H, Yoshida T, et al. Validation of grading scale for evaluating symptoms of idiopathic normal-pressure hydrocephalus. Dement Geriatr Cogn Disord. 2008;25(1):37-45. doi:10.1159/000111149

32. Liu CY, Gao J, Mao CH, et al. [The walking ability and cognitive function changing in normal pressure hydrocephalus patients after cerebrospinal fluid tap test]. Zhong Hua Shen Jing Ke Za Zhi. 2016;49(2): 15.(in Chinese) 
33. Virhammar J1, Cesarini KG, Laurell K.The CSF tap test in normal pressure hydrocephalus: evaluation time, reliability and the influence of pain. Eur J Neurol. 2012;19(2):271-6. doi: 10.1111/j.14681331.2011.03486.x.

34. Liu CY, Dong LL, Mao CH, et al. Correlation between white matter leisions and clinical features of patients with idiopathic normal pressure hydrocephalus in CSF tap test. Alzheimer's \& Dementia, the journal of Alzheimer's Association. 2019;15(7S):711. doi.org/10.1016/j.jalz.2019.06.2732

35. Liouta E, Gatzonis S, Kalamatianos T, et al. Finger tapping and verbal fluency post-tap test improvement in INPH: its value in differential diagnosis and shunt-treatment outcomes prognosis. Acta Neurochir (Wien). 2017;159(12):2301-2307. doi: 10.1007/s00701-017-3301-2.

36. Beauchet $\mathrm{O}$, Annweiler $\mathrm{C}$, Montero-Odasso M, et al. Gait control: a specific subdomain of executive function? J Neuroeng Rehabil. 2012;9(9):12. doi: 10.1186/1743-0003-9-12.

37. Tullberg M, Jensen C, Ekholm S, et al. Normal pressure hydrocephalus: vascular white matter changes on MR images must not exclude patients from shunt surgery.AJNR Am J Neuroradiol. 2001;22(9):1665-73.

\section{Figures}

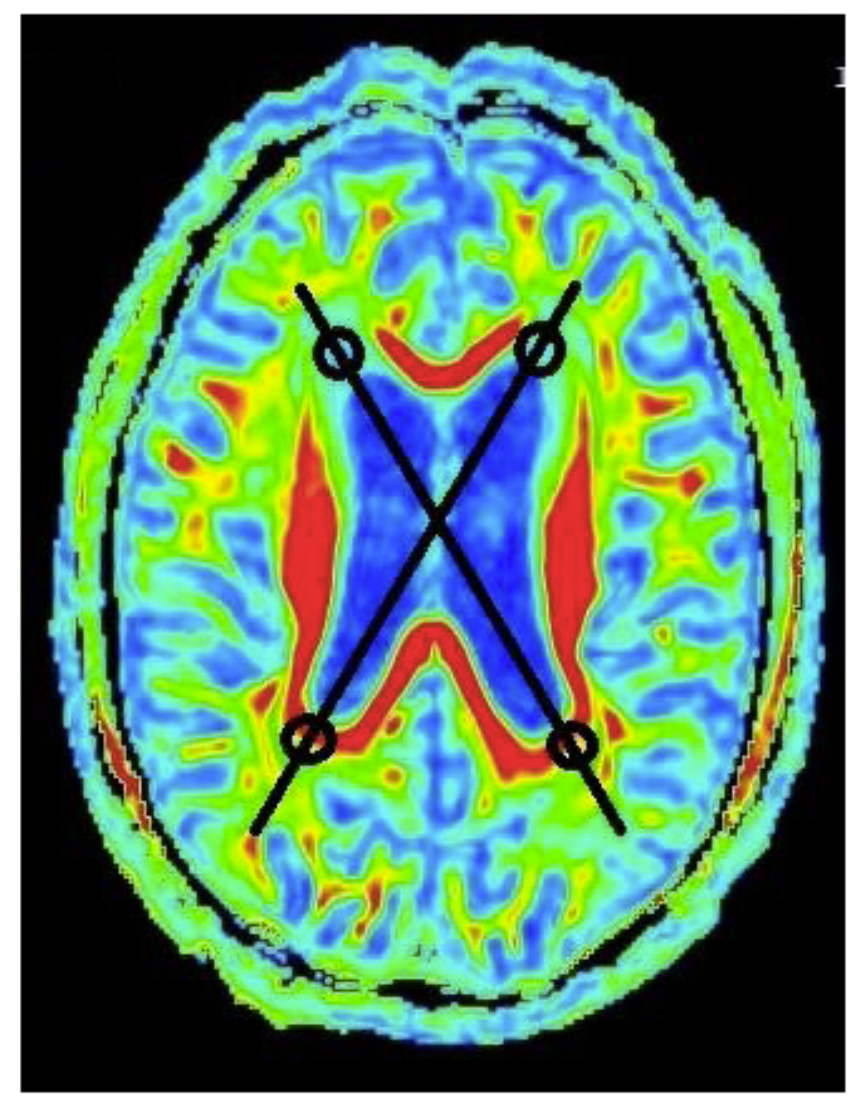

\section{Figure 1}

Diffusion tensor imaging illustrates the location of the regions of interest (ROIs). ROIs were the bilateral anterior and posterior periventricular white matter areas that were set as circular areas with a diameter of 2 
$\mathrm{mm}$ perpendicular to the longitudinal axis of the ipsilateral ventricle on the brain imaging slice with the lateral ventricle.

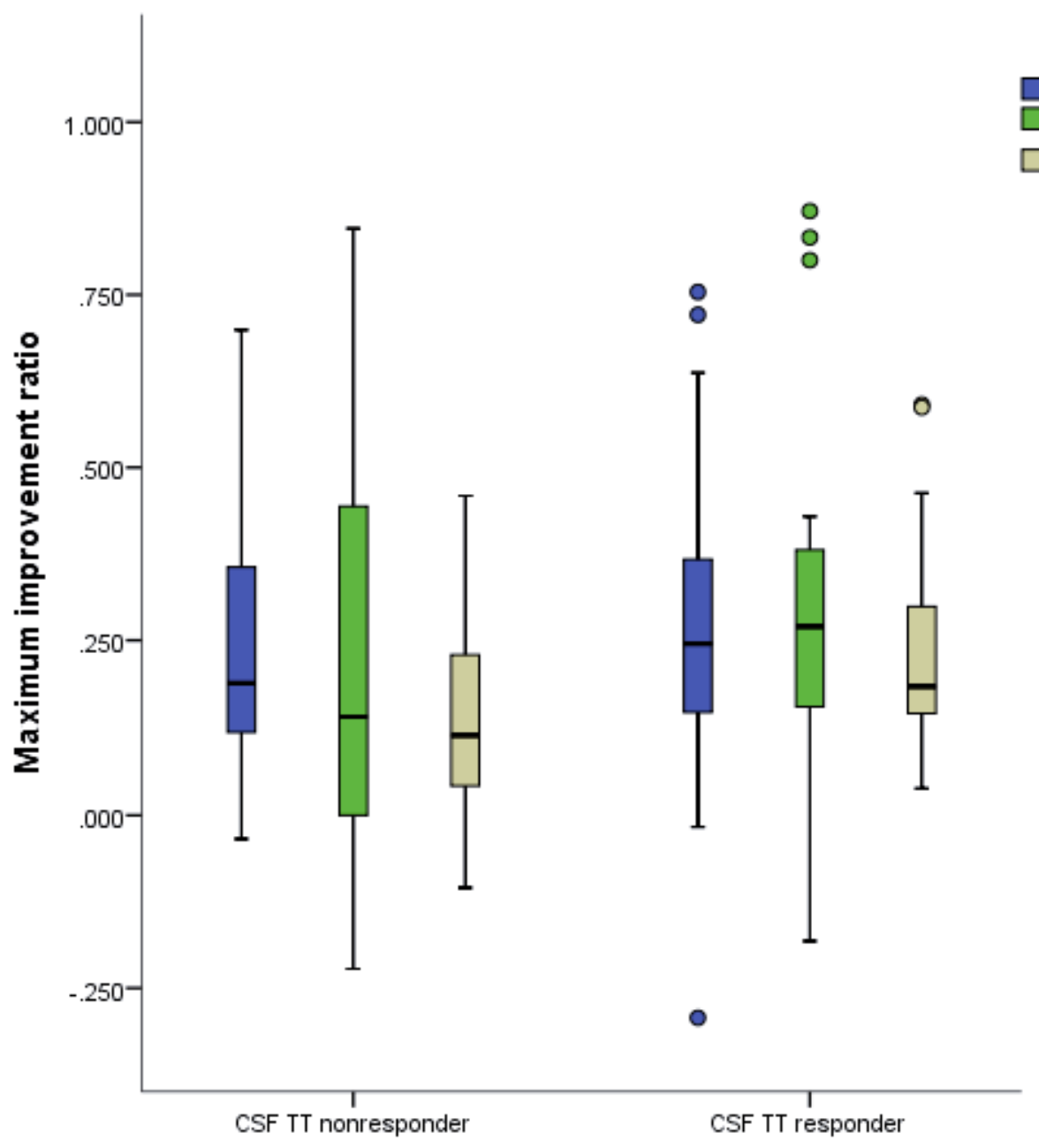

Figure 2

Difference in the maximum improvement ratio of the upper limb-related tests between the CSF TT responder and nonresponder groups. There was a significant difference in the maximum improvement ratio of the complex visual motor speed index between the two groups $(P<0.05)$, while the maximum improvement ratios for the grooved pegboard test and the symbol-digit modalities test were not different. 